\title{
Course of Hyposmia and Hypogeusia and their Relationship with Severity of COVID-19 Disease among Indian Population
}

\author{
Santhanakrishnan Kaliavaradan ${ }^{1} \cdot$ Poornima S. Bhat $^{1}\left(\mathbb{D} \cdot\right.$ Mariappan Rajagopal $^{1} \cdot$ \\ V. Vinayagamoorthy ${ }^{2} \cdot$ M. Vimal ${ }^{3} \cdot$ Sathiyanarayanan Janakiraman $^{4}$. \\ Nisha Muruganidhi $^{1} \cdot$ K. R. Srinivasane ${ }^{1} \cdot$ J. Jaswanthkumar $^{5} \cdot$ J. Ilamparithi $^{5}$
}

Received: 1 April 2021/Accepted: 20 June 2021/Published online: 10 August 2021

(C) Association of Otolaryngologists of India 2021

\begin{abstract}
Aims: Hyposmia and hypogeusia are one of the symptoms of COVID-19. Occurrence and course of these symptoms and their relationship with severity of COVID19 disease are studied. Materials and Methods: This was a prospective cohort study, including consenting adult SARS CoV-2 positive patients of both genders, admitted to a Covid Hospital in Puducherry, India. This questionnairebased study was conducted for a period 4 months from 1st October 2020 to 31st January 2021, and collected data was
\end{abstract}

Poornima S. Bhat

poornimasbhat86@gmail.com

Santhanakrishnan Kaliavaradan

santhanakrishnan2709@gmail.com

Mariappan Rajagopal

doctorrgm@gmail.com

V. Vinayagamoorthy

drvinayagamoorthy@gmail.com

M. Vimal

drvimalm@gmail.com

Sathiyanarayanan Janakiraman

drjsathiyanarayanan@gmail.com

Nisha Muruganidhi

nishamuruganidhi@gmail.com

K. R. Srinivasane

srinivasanekr@gmail.com

J. Jaswanthkumar

jjaswanthkumar1@gmail.com

J. Ilamparithi

ilamparithi03@gmail.com

1 Department of Otorhinolaryngology, Sri Manakula

Vinayagar Medical College and Hospital,

Kalitheerthalkuppam, Madagadipet, Puducherry 605107,

India analyzed using SPSS version 24.0 software. Results: Out of 639 participants, $412(64.5 \%)$ were males, $227(35.5 \%)$ were females. Total cases of new onset hyposmia were 167 $(26.1 \%)$, and total patients with new onset hypogeusia were 172 (26.91\%). 216 (33.80\%) had either hyposmia/hypogeusia. First symptom as hyposmia was noted in 49 $(7.67 \%)$ patients, and as hypogeusia in $20(3.13 \%)$ patients before development of any other symptoms. 216 (33.80\%) patients had either smell or taste disturbance as one of their

2 Department of Community Medicine, Sri Manakula Vinayagar Medical College and Hospital,

Kalitheerthalkuppam, Madagadipet, Puducherry 605107, India

3 Department of Pathology, Sri Manakula Vinayagar Medical College and Hospital, Kalitheerthalkuppam, Madagadipet, Puducherry 605107, India

4 Department of General Medicine, Sri Manakula Vinayagar Medical College and Hospital, Kalitheerthalkuppam, Madagadipet, Puducherry 605107, India

5 CRRI, Sri Manakula Vinayagar Medical College and Hospital, Kalitheerthalkuppam, Madagadipet, Puducherry 605107, India 
symptoms. By the end of 5 weeks of illness, $96.41 \%$ of hyposmic patients, and $97.67 \%$ of hypogeusic patients recovered fully. There was no statistically significant difference between presence or absence of hyposmia/hypogeusia and severity of COVID-19 disease ( $p$ value $=0.95)$. Conclusion: The occurrence of hyposmia and hypogeusia among Indian COVID-19 patients is more than $26 \%$. Presence or absence of hyposmia/hypogeusia is not a predictor of severity of COVID-19 disease. More than $96 \%$ of the patients fully recovered their sense of smell and taste sensation by the end of 5 weeks.

Keywords Hyposmia - Hypogeusia COVID-19 . Symptom $\cdot$ Severity

\section{Introduction}

Most patients infected with SARS-CoV-2 experience a mild disease. In the most severe cases, nearly $5 \%$ patients may develop pneumonia, acute respiratory failure, distress syndrome, and acute heart problems, multiorgan failure. [1-3] The reports of olfactory dysfunction in otherwise asymptomatic persons have led to increased interest in this sign as a potential early indicator of SARS-CoV-2 infection $[4,5]$.

World Health Organization (WHO), declared COVID19 as pandemic in March 2020 [6]. People with asymptomatic or with unrecognised manifestations of the disease are also pointed as the virus main transmission source [7]. There are some studies which implicated that loss of smell as an independent positive prognostic factor of a less severe COVID-19 infection, associated with decreased hospitalization, intensive care unit admissionand severe stage of COVID-19 infection. [8, 9]

Based on our systematic literature search, we found out that, there are very few literatures on the course of these two symptoms of COVID-19 in Indian population. The onset, duration and recovery of new onset hyposmia and/or hypogeusia and their relationship to the severity of COVID-19 disease are still unclear in our country. This study was conducted to estimate the proportion of COVID19 positive patients presenting with the symptoms of hyposmia and hypogeusia as first and/or one of the symptoms, and to study the course of hyposmia and hypogeusia and to find out any correlation between hyposmia and hypogeusia with the severity of COVID-19 infection among Indian population.

\section{Materials and Methods}

\section{Study Setting and Study Design}

The study was done by the Department of Otorhinolaryngology and Head and Neck surgery, in the Covid ward of a tertiary care teaching hospital located at rural part of Puducherry, a union territory in Southern India. Our hospital is a Dedicated Covid Hospital (DCH) that was approved by the Health department of Puducherry. The study was approved by the Institutional Ethics Committee. (IEC No.49/2020). A written, informed consent was obtained from all the participants. This was a prospective type of cohort study. The status of smell and taste disturbances were ascertained at the time of admission and their course, and the details of outcome of SARS-CoV2 infection were collected prospectively.

\section{Study Participants, Sample Size and Sampling}

Patients who were at least 18 years of age, of any gender, tested positive for SARS-CoV-2 viral infection by RT-PCR and admitted in our hospital were the study participants. Those patients who were known case of chronic smell or taste disturbance and those with severe stage of Covid-19 infection were excluded from the study. The sample size was calculated to be atleast 415 , using OpenEpi software, version 3.2 taking into consideration $33.9 \%$ of patients with loss of smell among hospitalized COVID-19 patients, with $95 \%$ confidence interval, $80 \%$ power and $20 \%$ loss to follow up [10]. However all eligible patients during the study period was included, and final sample size of 639 was achieved.

\section{Data Collection}

Data was collected for a period of four months from 1st October 2020 to 31st January 2021. Pre-structured and pilot tested questionnaire was used to collect the information. It had the following sections. First section had sociodemographic details, second had information on symptoms of Covid-19 infection that included fever, cough, breathlessness, loss of smell, loss of taste, body pain, tiredness, nausea/vomiting, diarrhoea, throat pain and headache. The order of appearance of these symptoms one week before and four weeks after the test result was noted. If patients had given history of hyposmia and hypogeusia, they were asked to mark their self-perceived severity using a Likert scale that ranged from 0 to 4 , indicating normal, minimal loss, moderate loss, severe loss, and totally absent. History of any altered sensation was also obtained. All the personal protective measures were strictly followed throughout the 
data collection procedure. Patients who were discharged from the hospital were followed up over telephone weekly once till five weeks. The severity of the COVID-19 disease of the patient at presentation and during the hospital stay were noted down from the case records, based on the interim guidance of $\mathrm{WHO}$ on clinical management of COVID-19 as mild, moderate, severe, critical disease.

\section{Statistical Analysis}

Collected data were digitally entered in an excel sheet and was analyzed using SPSS version 24.0 analytical software. Frequency and percentage were used to describe the categorical variables. The proportion of hyposmia among study participants was reported with its $95 \%$ confidence interval. Relative risk was calculated to ascertain the strength of association between the hyposmia/hypogeusia status and the outcome.

\section{Results}

A total of 639 patients were included in this study after excluding patients with chronic smell or taste disturbances. Out of these, $412(64.5 \%)$ were males, 227 (35.5\%) were females. Total cases of hyposmia were 167 (26.1\%); 95\% CI: 22.8-29.6, and total patients with hypogeusia were 172 (26.9\%); 95\% CI: 23.6-30.5.Among 412 male patients, $103(25 \%)$ had loss of smell, and among total of 227 female patients, 64 (28.19\%) had loss of smell.

Majority of the patients belonged to the age group between 36 and 65 years i.e. 407 (63.7\%), followed by 18-35 years i.e., $172(26.9 \%)$, only 60 (9.4\%) were above 65 years. Majority of the patients were employed i.e., 401 $(62.8 \%), 196(30.7 \%)$ were unemployed, and 42 (6.6\%) were students.

49 (7.67\%) patients had loss of smell as their first symptom, and $20(3.13 \%)$ of the patients presented with loss of taste as their first symptom, before development of any other symptoms. 216 (33.80\%) patients had either smell or taste disturbance as one of their symptoms. 49 (7.67\%) patients had taste disturbances without any smell disturbances.

\section{Course of Self-Perceived of Severity of Loss of Smell among Hyposmic Patients $(n=167)$ Over the Weeks of Illness (Table 1)}

Out of 639 patients, 167 (26.1\%) had smell disturbances during the first week of illness, with majority of them having minimal loss 80 (47.9\%), followed by moderate loss $39(23.35 \%)$, severe loss 25 (14.97\%) and complete loss 20 $(11.97 \%)$. Three $(1.79 \%)$ patients also had altered smell sensation. By the end of 1 week, 56 (33.53\%) patients had complete resolution of smell disturbances. During second week of illness, $60(35.92 \%)$ patients had minimal loss of smell, followed by moderate loss in $28(16.76 \%)$, severe loss $13(7.78 \%)$ and complete loss in nine (5.39\%) patients. One $(0.59 \%)$ patient had altered smell sensation. During third week of illness, minimal loss was noted in $72(43.11 \%)$ patients, followed by moderate loss in nine $(5.39 \%)$ patients, severe loss in three $(1.79 \%)$ patients and complete loss in one $(0.59 \%)$ patient. No patients had altered smell sensation. During fourth week of illness, majority had minimal loss 20 (11.97\%), followed by moderate loss in three $(1.79 \%)$ patients and complete loss in one $(0.59 \%)$ patient. During 5 week of illness, five (2.99\%) patients had minimal loss of smell and one $(0.59 \%)$ had moderate loss of smell.

\section{Course of Self-perceived Severity of Loss of Taste among Hypogeusic Patients $(n=172)$ Over the Weeks of Illness (Table 2)}

$172(26.91 \%)$ out of 639 patients had taste disturbances during the 1 week of illness, with majority of them having minimal loss of taste i.e., 85 (49.41\%), followed by moderate loss in $49(28.49 \%)$, severe loss in $18(10.46 \%)$ and complete loss in 18 (10.46\%). 2 (1.16\%) patients had altered taste sensation. During 2 week of illness, 98 (56.97\%) patients had minimal loss of taste, followed by moderate loss in $36(20.93 \%)$ patients, severe loss in 12 $(6.97 \%)$ and complete loss in $6(3.49 \%)$. Two $(1.16 \%)$ patients had altered taste sensation. During 3 week of illness, majority of patients had minimal loss i.e., 72 $(41.86 \%)$ followed by moderate loss in $10(5.81 \%)$ patients, severe loss in one $(0.58 \%)$ patient and complete loss in two $(1.16 \%)$ patients. one $(0.58 \%)$ patient had altered taste sensation. During 4 week of illness, 21 (12.2\%) patients had minimal loss of taste, followed by moderate loss in 9 (5.23\%) patients. During fifth week of illness, three $(1.74 \%)$ patients had minimal loss and one $(0.58 \%)$ patient had moderate loss of taste.

\section{Comparison between Patients With and Without Hyposmia/Hypogeusia with Respect to Supplementary Oxygen Requirement /ICU Admission (Table 3)}

Among total of 639 patients, 216 (33.80\%) had either smell or taste disturbances as one of their symptoms. Among these patients, six $(2.77 \%)$ patients required supplemental $\mathrm{O}_{2}$ support/ICU admission wheareas 210 (97.23\%) did not require any. $423(66.2 \%)$ out of total 639 patients did not have any smell or taste disturbances. Among these patients, $12(2.84 \%)$ required supplemental Oxygen $\left(\mathrm{O}_{2}\right) / \mathrm{ICU}$ admission whereas $411(97.16 \%)$ did not require any. The 
Table 1 Course of Self-perceived severity of loss of smell among hyposmic patients $(n=167)$ over 5 weeks of illness

\begin{tabular}{lccccc}
\hline $\begin{array}{l}\text { Self-perceived severity of loss of smell among } \\
\text { hyposmic patients }\end{array}$ & $\begin{array}{l}\text { 1st week of } \\
\text { illness n (\%) }\end{array}$ & $\begin{array}{l}\text { 2nd week of } \\
\text { illness n (\%) }\end{array}$ & $\begin{array}{l}\text { 3rd week of } \\
\text { illness n }(\%)\end{array}$ & $\begin{array}{l}\text { 4th week of } \\
\text { illness n }(\%)\end{array}$ & $\begin{array}{l}\text { 5th week of } \\
\text { illness n }(\%)\end{array}$ \\
\hline 1 (minimal loss) & $80(47.9 \%)$ & $60(35.92 \%)$ & $72(43.11 \%)$ & $20(11.97 \%)$ & $5(2.99 \%)$ \\
2 (moderate loss) & $39(23.35 \%)$ & $28(16.76 \%)$ & $9(5.39 \%)$ & $3(1.79 \%)$ & $1(0.59 \%)$ \\
3 (severe loss) & $25(14.97 \%)$ & $13(7.78 \%)$ & $3(1.79 \%)$ & $00 \%)$ & $0(0 \%)$ \\
4 (complete loss) & $20(11.97 \%)$ & $9(5.39 \%)$ & $1(0.59 \%)$ & $1(0.59 \%)$ & $0(0 \%)$ \\
Altered smell & $3(1.79 \%)$ & $1(0.59 \%)$ & $0(0 \%)$ & $0(0 \%)$ & $0(0 \%)$ \\
\hline
\end{tabular}

Table 2 Course of Self perceived severity of loss of taste among hypogeusic patients $(\mathrm{n}=172)$ over 5 weeks of illness

\begin{tabular}{lccccc}
\hline $\begin{array}{l}\text { Self perceived severity of loss of taste among } \\
\text { hypogeusic patients }\end{array}$ & $\begin{array}{l}\text { 1st week of } \\
\text { illness n }(\%)\end{array}$ & $\begin{array}{l}\text { 2nd week of } \\
\text { illness n }(\%)\end{array}$ & $\begin{array}{l}\text { 3rd week of } \\
\text { illness n }(\%)\end{array}$ & $\begin{array}{l}\text { 4th week of } \\
\text { illness n }(\%)\end{array}$ & $\begin{array}{l}\text { 5th week of } \\
\text { illness n }(\%)\end{array}$ \\
\hline 1 (minimal loss) & $85(49.41 \%)$ & $98(56.97 \%)$ & $72(41.86 \%)$ & $21(12.2 \%)$ & $3(1.74 \%)$ \\
2 (moderate loss) & $49(28.49 \%)$ & $36(20.93 \%)$ & $10(5.81 \%)$ & $9(5.23 \%)$ & $1(0.58 \%)$ \\
3 (severe loss) & $18(10.46 \%)$ & $12(6.97 \%)$ & $1(0.58 \%)$ & 0 & 0 \\
4 (complete loss) & $18(10.46 \%)$ & $6(3.49 \%)$ & $2(1.16 \%)$ & 0 & 0 \\
Altered taste & $2(1.16 \%)$ & $2(1.16 \%)$ & $1(0.58 \%)$ & 0 & 0 \\
\hline
\end{tabular}

Table 3 Comparison between patients with and without hyposmia/hypogeusia with respect to supplementary Oxygen requirement/ICU admission

\begin{tabular}{lccc}
\hline Hyposmia/hypogeusia & \multicolumn{1}{c}{$\mathrm{O}_{2}$ supplementation/ICU admission required } & Relative risk(95\% ci) \\
\cline { 2 - 4 } & Yes n $(\%)$ & No n $(\%)$ & $0.97(0.4-2.6)$ \\
\hline Yes & $6(2.77)$ & $210(97.23)$ & \\
No & $12(2.84)$ & $411(97.16)$ & 0.95 \\
\hline
\end{tabular}

ci-confidence interval, $\wedge p$ value based on chi-square test

relative risk was 0.97 and the difference between the two groups was statistically not significant $(p$ value $=0.95)$.

\section{Analysis of Association between Hyposmial Hypogeusia with Respect to Gender and Age Groups (Table 4)}

Occurrence of hyposmia/hypogeusia based on gender was assessed using Chi-Square test, and it was found that

Table 4 Analysis of association between hyposmia/hypogeusia with respect to gender and age groups

\begin{tabular}{|c|c|c|c|c|c|c|}
\hline \multicolumn{2}{|c|}{ Socio-demographic features $(N=639)$} & \multirow[t]{2}{*}{ Number (\%) } & \multicolumn{2}{|c|}{ Smell disturbance $\mathrm{n}(\%)^{\#}$, } & \multirow[t]{2}{*}{ RR $(95 \%$ CI) } & \multirow[t]{2}{*}{$p$ value $^{\wedge}$} \\
\hline & & & Yes & No & & \\
\hline \multirow[t]{2}{*}{ Gender } & Female & $227(35.5)$ & $64(28.2)$ & $163(71.8)$ & $1.1(0.9-1.5)$ & 0.37 \\
\hline & Male & $412(64.5)$ & $103(25)$ & $309(75)$ & 1 (Reference) & \\
\hline \multirow[t]{3}{*}{ Age group in Years } & $18-35$ & $172(26.9)$ & $65(37.8)$ & $107(62.2)$ & $3.2(1.6-6.7)$ & $<0.001 *$ \\
\hline & $36-65$ & 407 (63.7) & $95(23.3)$ & $312(76.7)$ & $2(0.9-4.1)$ & 0.06 \\
\hline & $>65$ & $60(9.4)$ & $7(11.7)$ & $53(88.3)$ & 1 (Reference) & NA \\
\hline
\end{tabular}

@ column $\%$, \# row \%,CI-confidence interval, $N A$-not applicable, $R R$ - Relative Risk ${ }^{\wedge} p$ value based on chi-square test, $* p$ value statistically significant $(<0.05)$ 
relative risk was 1.1 , slightly more for females, but it was not statistically significant $(p$ value $=3.7)$. (Table 4) Among the age groups, young patients in 18-35 years of age group are at statistically significant $(\mathrm{RR}=3.2$, $p$ value $<0.001$ ) higher risk of having hyposmia/hypogeusia. Patients in the age group 36-65 years are also at higher risk $(\mathrm{RR}=2)$, but it was not statistically significant. Patients older than 65 years did not show any difference.

\section{Discussion}

Anosmia and aguesia have been identified as one of the early symptoms of COVID-19. Multiple cross-sectional studies have demonstrated that the incidence rate of olfactory dysfunction in COVID-19 patients varies from 33.9 to $68 \%$ with female dominance [11]. In our study, the occurrence of loss of smell was almost similar, with slightly higher female dominance, but it was not statistically significant.In a study including 214 hospitalized COVID-19 patients in Wuhan, 5.1\% and 5.6\% of patients presented with hyposmia and hypogeusia, respectively [12]. More recent olfactory surveys on COVID-19 patients showed olfactory dysfunction in 20-85\% of patients.[10, 13]. In another case report, sudden onset of anosmia as well as ageusia was noted as the first symptom of COVID [14]. In our study, $7.67 \%$ patients had loss of smell as their first symptom, and $3.13 \%$ of the patients presented with loss of taste as their first symptom, before development of any other symptoms.

In a recent cohort study done on 382 COVID-19 positive patients with anosmia, an online survey was conducted at interval of 1 week. Among them, 86.4\% reported complete anosmia and a further $11.5 \%$ a very severe loss of smell at the time of completing the first survey week. At follow-up 1 week later, there was significant improvement in selfrating of severity of olfactory loss [15]. In another study done in Belgium, observed that first 2 months, $79.5 \%$ of patients may expect to have complete recovery of their olfactory function. The severity of olfactory loss may predict the lack of mid-term recovery [16]. There are very few literatures on the trend of anosmia and ageusia in Indian population. In a recent study done in India, it was noted that the prevalence of new onset anosmia in Indian population with COVID-19 is $14.8 \%$, which was much lesser than what was reported in European population. All the patients had regained their sense of smell at the end of 3 weeks [17]. In our study, out of total 639 patients, the occurence of smell disturbances was $26.13 \%$ and occurence of taste disturbances was $26.9 \%$. During the first week of illness, out of 167 patients who who had some smell disturbances, $47.9 \%$ had minimal loss, $23.35 \%$ had moderate loss, $14.97 \%$ patients had severe loss of smell,
$11.97 \%$ patients had complete loss of smell, whereas $1.79 \%$ had altered smell sensation. Patients improved progressively and by the end of 5 th week, $2.99 \%$ patients had minimal loss of smell and $0.6 \%$ patient had moderate loss of smell. Rest all $96.41 \%$ patients had normal smell sensation at the end of 5 weeks.

During the first week of illness, out of 172 patients who had some taste disturbances, $49.42 \%$ patients had minimal loss, $28.49 \%$ patients had moderate loss, $10.46 \%$ patients had severe loss of smell, $10.46 \%$ patients had complete loss of taste, whereas $1.16 \%$ had altered taste sensation. Patients improved progressively and by the end of 5th week, $1.74 \%$ patients had minimal loss of taste and $0.58 \%$ patients had moderate loss of taste. Rest all $97.67 \%$ patients had recovered with normal taste sensation by the end of 5 weeks.

Only hypogeusia has also been reported as the first symptom of COVID-19. In a case report of a 59 years old female, hypoguesea was the first clinical symptom of COVID, progressing with anosmia, fever and respiratory symptoms, requiring ICU admission, later, recovery and discharge with mild loss of taste at day 16 after the symptom onset [18]. In our study, $3.13 \%$ of the patients presented with loss of taste as their first symptom and $7.67 \%$ patients had taste disturbances without any smell disturbances.

There are very few studies which have tried to explore the relationship between the anosmia and the severity of Covid-19 infection. Majority of these studies have concluded that anosmia is associated with less severe Covid-19 disease [8, 9]. A recent study done in Spain noted that hospitalized Covid-19 patients with anosmia had a lower adjusted mortality rate and less severe course of the disease [19]. While these findings are very assuring, this might give a false sense of safety to the patients and treating clinicians, especially in developing countries like India, where people with anosmia/ageusia may not report to hospitals for COVID-19 testing, believing that it will cause only minor disease, even if they are SARS-COV-2 positive. They may continue with their routine daily activities, without taking precautions, thus contributing to the spread of pandemic. There is limited literature on relationship between hyposmia and severity OF COVID-19 among Indian population. In contrary to majority of these studies, in our study it was noted that, there was no statistically significant difference between patients progressing to severe stage of COVID-19 requiring supplemental O2/ICU admissions and presence or absence of hyposmia/hypogeusia. This was similar to a study conducted in Germany which noted that anosmia is not a predictor of a severe COVID-19 manifestation [20].

Hence, the course of new onset hyposmia and hypogeusia in COVID-19 patients in Indian population is 
similar to most of the studies in the world, with majority of the patients recovering completely by $4-5$ weeks. But there is no statistically significant difference in the severity of COVID-19 disease and presence or absence of smell and taste disturbances.

Strengths of this study is that it was a prospective study with large sample size compared to previous studies on Indian populations addressing the hyposmia/hypogeusia symptoms. Course of severity of loss of smell and taste was studied over the weeks. Smell and taste disturbance as first symptom was also noted. Proportion of taste disturbances without any smell symptoms was also noted. To the best of our knowledge, evaluating the relationship between smell and taste disturbances with severity of COVID-19 infection among Indian population was not reported before. Appropriate statistical methods were used to analyse the data. Loss to follow up was meticulously reduced by giving proper instruction at the time of discharge and following them up telephonically during their convenient time.

Limitations of this study is that, only subjective severity of the smell and taste sensation was noted and correlation between the medications and the smell/taste disturbances was not studied. Data collected from hospital-setting could not allow us to calculate incidence of the anosmia and ageusia in general population.

\section{Conclusion}

The occurrence of hyposmia among Indian COVID-19 patients is $26.1 \%$ and that of hypogeusia is $26.8 \%$. The proportion of patients presenting with hyposmia as the first symptom is $7.67 \%$ and hypogeusia as first symptom is $3.13 \%$. There was no statistically significant difference between presence or absence of hyposmia/hypogeusia and severity of stage of COVID-19 disease. More than $96 \%$ of the patients fully recovered their sense of smell and taste sensation by the end of 5 weeks. Increased public awareness measures regarding these symptoms and its prognosis are recommended, which can help in early diagnosis, isolation and prevention of spread of pandemic. It should be highlighted to the patients and clinicians that the hyposmia and hypogeusia are neither predictors nor protective of severity of COVID-19 disease.

Acknowledgements We thank all the faculties, Postgraduate residents, interns, nursing staff and all the other staff members who are working in our COVID wards risking their lives. We thank the Epidemiology department of Community Medicine, for their support.

Funding No funds, grants, or other support was received.

Availability of data and material Available.

\section{Declarations}

Conflict of interest The authors have no conflict of interest to declare that are relevant to the content of this article.

Ethical approval This study involved human participants and was approved by the institutional ethics committee [Sri Manakula Vinayagar Medical College and Hospital-Ethics committee (Human studies), No.EC/49/2020].

Informed consent Informed consent was obtained from all individual participants included in the study.

\section{References}

1. Weiss P, Murdoch D (2020) Clinical course and mortality risk of severe COVID-19. The Lancet 395(10229):1014-1015

2. Zhou F, Yu T, Du R, Fan G, Liu Y, Liu Z et al (2020) Clinical course and risk factors for mortality of adult in-patients with COVID-19 in Wuhan, China: a retrospective cohort study. Lancet 395(10229):1054-1062

3. Wu Z, McGoogan J (2020) Characteristics of and important lessons from the Coronavirus disease 2019 (COVID-19) outbreak in China. JAMA 323(13):1239. https://doi.org/10.1001/jama.2020.26

4. Hopkins C, Surda P, Kumar N (2020) Presentation of new onset anosmia during the COVID-19 pandemic. Rhinol J 58(3):295-298

5. Gane S, Kelly C, Hopkins C (2020) Isolated sudden onset anosmia in COVID-19 infection. A Novel Syndrome? Rhinol J 58(3):299-301

6. Bridwell R, Long B, Gottlieb M (2020) Neurologic complications of COVID-19. Am J Emerg Med 38:1549

7. Bai Y, Yao L, Wei T, Tian F, Jin D, Chen L et al (2020) Presumed asymptomatic carrier transmission of COVID-19. JAMA 323(14): 1406

8. Foster K, Jauregui E, Tajudeen B, Bishehsari F, Mahdavinia M (2020) Smell loss is a prognostic factor for lower severity of coronavirus disease 2019. Ann Allergy Asthma Immunol 125(4):481-483. https://doi.org/10.1016/j.anai.2020.07.023

9. Yan C, Faraji F, Prajapati D, Ostrander B, DeConde A (2020) Self-reported olfactory loss associates with outpatient clinical course in COVID-19. Int Forum Allergy Rhinol 10(7):821-831

10. Giacomelli A, Pezzati L, Conti F, Bernacchia D, Siano M, Oreni L et al (2020) Self-reported olfactory and taste disorders in patients with severe acute respiratory Coronavirus 2 infection: a cross-sectional study. Clin Infect Dis 71(15):889-890. https://doi.org/10.1093/cid/ciaa330

11. Meng X, Deng Y, Dai Z, Meng Z (2020) COVID-19 and anosmia: A review based on up-to-date knowledge. Am J Otolaryngol 41(5):102581. https://doi.org/10.1016/j.amjoto.2020.102581

12. Mao L, Jin H, Wang M, Hu Y, Chen S, He Q et al (2020) Neurologic manifestations of hospitalized patients With Coronavirus disease 2019 in Wuhan, China. JAMA Neurol 77(6):683

13. Lechien J, Chiesa-Estomba C, De Siati D, Horoi M, Le Bon S, Rodriguez A et al (2020) Olfactory and gustatory dysfunctions as a clinical presentation of mild-to-moderate forms of the coronavirus disease (COVID-19): a multicenter European study. Eur Arch Otorhinolaryngol 277(8):2251-2261. https://doi.org/10.1007/500405-020

14. Daher V, Soares Oliveira D, Daher Júnior M, de Melo Júnior, Fernandes E, Bomtempo de Castro J, Moya M et al (2020) Anosmia: a marker of infection by the new corona virus. Resp Med Case Rep 31:101129 
15. Hopkins C, Surda P, Whitehead E, Kumar B (2020) Early recovery following new onset anosmia during the COVID-19 pandemic-An observational cohort study. J Otolaryngol-Head Neck Surg. https://doi.org/10.1186/s40463-020-00423-8

16. Lechien JR, Journe F, Hans S et al (2020) Severity of anosmia as an early symptom of COVID-19 infection may predict lasting loss of smell. Front Med (Lausanne) 7:582802. https://doi.org/10.3389/fmed.2020.582802

17. Mishra P, Gowda V, Dixit S, Kaushik M (2020) prevalence of new onset anosmia in COVID-19 Patients: Is The trend different between European and Indian population? Indian J Otolaryngol Head Neck Surg. https://doi.org/10.1007/s12070-020-01986-8

18. Melley L, Bress E, Polan E (2020) Hypogeusia as the initial presenting symptom of COVID-19. BMJ Case Rep 13(5):e236080. https://doi.org/10.1136/bcr-2020-236080
19. Talavera B, García-Azorín D, Martínez-Pías E, Trigo J, Hernández-Pérez I, Valle-Peñacoba G et al (2020) Anosmia is associated with lower in-hospital mortality in COVID-19. J Neurol Sci 15(419):117163. https://doi.org/10.1016/j.jns.2020.117163

20. Hornuss D, Lange B, Schröter N, Rieg S, Kern WV, Wagner D (2020) Anosmia in COVID-19 patients. Clin Microbiol Infect 26(10):1426-1427. https://doi.org/10.1016/j.cmi.2020.05.017

Publisher's Note Springer Nature remains neutral with regard to jurisdictional claims in published maps and institutional affiliations. 